\title{
Review Article \\ Regulation of Immune Cell Functions by Metabolic Reprogramming
}

\author{
Jaehong $\operatorname{Kim} \mathbb{D}^{1,2}$ \\ ${ }^{1}$ Department of Biochemistry, School of Medicine, Gachon University, Incheon 21999, Republic of Korea \\ ${ }^{2}$ Department of Health Sciences and Technology, Gachon Advanced Institute for Health Science and Technology, Gachon University, \\ Incheon 21999, Republic of Korea
}

Correspondence should be addressed to Jaehong Kim; geretics@gachon.ac.kr

Received 19 October 2017; Accepted 14 January 2018; Published 13 February 2018

Academic Editor: Abdallah Elkhal

Copyright ( 2018 Jaehong Kim. This is an open access article distributed under the Creative Commons Attribution License, which permits unrestricted use, distribution, and reproduction in any medium, provided the original work is properly cited.

Recent findings show that the metabolic status of immune cells can determine immune responses. Metabolic reprogramming between aerobic glycolysis and oxidative phosphorylation, previously speculated as exclusively observable in cancer cells, exists in various types of immune and stromal cells in many different pathological conditions other than cancer. The microenvironments of cancer, obese adipose, and wound-repairing tissues share common features of inflammatory reactions. In addition, the metabolic changes in macrophages and $\mathrm{T}$ cells are now regarded as crucial for the functional plasticity of the immune cells and responsible for the progression and regression of many pathological processes, notably cancer. It is possible that metabolic changes in the microenvironment induced by other cellular components are responsible for the functional plasticity of immune cells. This review explores the molecular mechanisms responsible for metabolic reprogramming in macrophages and $\mathrm{T}$ cells and also provides a summary of recent updates with regard to the functional modulation of the immune cells by metabolic changes in the microenvironment, notably the tumor microenvironment.

\section{Introduction}

Pleiotropic interactions between various cells are responsible for the maintenance and disturbance of homeostasis in the tissue microenvironment of physiological and pathological conditions. For example, from early carcinogenesis to progression and metastasis, cancer cells interact with various types of stromal cells, for example, cancer-associated fibroblasts, endothelial cells, and immune cells in the tumor microenvironment (TME). The TME is flooded with cytokines and growth factors responsible for "smoldering persistent inflammation." This reactive stroma is a wellcharacterized component of the TME that shows similarities to the repair response in injured tissue [1]. Recent findings revealed that various immune cell subsets are dominant regulators of the delicate balance between homeostasis and disturbance in the tissue microenvironment [2-5]. For example, macrophages can form a major component of immune cell infiltrate in the TME, constituting as much as half of a tumor mass [6, 7]. Immune responses of M1 and M2 macrophages describe the opposing activities of killing or repairing. The typical M1 macrophages drive inflammation and show high antigen presentation, high production of inflammatory cytokines such as IL-12 and IL-23, and high production of nitric oxide (NO) and reactive oxygen intermediates. In contrast, M2-type responses are the "resting" phenotype and are observed in the resolution of inflammation without infections, tissue remodeling, and repair. It has been widely accepted that IFN $\gamma$ alone or with microbial LPS or cytokines such as GM-CSF and TNF induces classically activated M1 macrophages, and IL-4, IL-6, IL-10, IL-13, IL-21, IL-33, immune complexes, and Notch can induce the M2 form of macrophage activation $[8,9]$. Notably, truly polarized macrophages are rare [10-13] and tumor-associated macrophages (TAMs) can be also described as M(IL-4), M(Ig), $\mathrm{M}(\mathrm{IL}-10), \mathrm{M}(\mathrm{GC}$ : glucocorticoid), $\mathrm{M}(\mathrm{IFN} \gamma), \mathrm{M}(\mathrm{LPS})$, and 
so forth, according to a recently attempted nomenclature based on specific activation standard [12]. Evidence supports a tumor-promoting role of TAMs, and high frequencies of TAMs are generally associated with poor prognosis in most human cancers $[2,14,15]$. TAMs infiltrating established tumors generally show the properties of an M2-like activated anti-inflammatory, protumoral properties rather than M1like activated proinflammatory, antitumoral phagocytic properties [16-18].

Macrophages can also form a major component of immune cell infiltrate in obese adipose tissue (AT), constituting as much as $40 \%$ of all AT cells [19]. In the progression of obesity, a switch from M2-like to M1-like activation of the macrophage population occurs and inflammatory cytokines such as tumor necrosis factor (TNF) contribute to insulin resistance in adipocytes characterized by an impaired insulin response such as hypertriglyceridemia and elevated fasting glucose $[20,21]$. In addition, lymphoid as well as myeloid cells infiltrates and expands in the liver tissue and the obese AT and these immune cell subsets are responsible for the development of obesityrelated metabolic dysregulation due to excessive nutrient intake and exacerbation of low-grade inflammatory changes in the microenvironment. $\mathrm{CD}^{+} \mathrm{T}$ cells also promote inflammation and metabolic disturbance in the AT [22]. In addition to macrophages and $\mathrm{T}$ cells, neutrophils and mast cells can also disturb the homeostasis in the tissue microenvironment.

Many recent findings in the field of immunometabolism now show that metabolic status in immune cells can determine various types of immune responses. Immune cells have remarkably diverse functions and cellular activities that are associated with distinct metabolic demands. The traditional simple concept of production of cellular ATP is that glycolysis generates two molecules of ATPs from one molecule of glucose. Glycolysis metabolizes glucose to pyruvate first, and the pyruvate is further metabolized to carbon dioxide, $\mathrm{NADH}$, and $\mathrm{FADH}_{2}$ in the mitochondria. The reducing equivalents ( $\mathrm{NADH}$ and $\mathrm{FADH}_{2}$ ) drive oxidative phosphorylation (OXPHOS) for more ATP synthesis. In the 1920s, it was demonstrated that cancer tissues can metabolize, even in aerobic conditions, about tenfolds more glucose to produce lactate than normal tissues can and this is known as aerobic glycolysis or the Warburg effect [23]. Since pyruvate is metabolized to lactate and secrete, lactate appears to be wasted in aerobic glycolysis. However, lactate secretion out of cells allows increased continuous glucose influx from the generation of $\mathrm{NAD}^{+}$and resultant accumulation of glycolytic intermediates facilitates biomass synthesis for rapidly proliferating cells. Since the observation and dramatic revitalization of the Warburg effect, the dominant glycolysis and relatively reduced OXPHOS were thought to be confined to cancer cells. However, recent findings clearly show that the Warburg effect-like metabolic reprogramming also exists in rapidly proliferating cells including various types of immune cells, most notably in macrophages and $\mathrm{T}$ cells, and determines the function of the immune cell subsets in disease conditions such as those in inflamed tissue or cancer [24-27].

\section{Metabolic Regulation of Macrophage Phenotypes}

The function of macrophages is not limited to the maintenance of homeostasis in the tissue microenvironment but also includes many activities such as cytokine production and phagocytosis upon their activation. Importantly, macrophages are famous for their plasticity and adoption of various activation states in response to their functional requirements signaled from their microenvironment. For example, an innate arm of the immune system can have an important capacity to adapt after challenged with pathogens [28]. This is known as innate immune memory or trained immunity. Trained immunity from epigenetic reprogramming of macrophages shows high glucose consumption and a high ratio of $\mathrm{NAD}^{+}$to its reduced form $\mathrm{NADH}$, reflecting a shift in metabolism with an increase in glycolysis and M1-like activation of macrophages, dependent on the activation of mTOR through the Akt-HIF-1 $\alpha$ pathway [29]. M2-like activated macrophages exploit fatty acid oxidation (FAO) to fuel OXPHOS rather than aerobic glycolysis for ATP production [30-32].

Of note, HIF1 $\alpha$ and $\mathrm{NF} \kappa \mathrm{B}$ drive the M1 phenotypes $[33,34]$ and PGC1 $\beta$, and peroxisome proliferator-activated receptors and STAT6 drive the M2 phenotypes (Figure 1) [35-38]. Phosphorylation and activation of a nutritional sensor, AMPK, regulate mitochondrial biogenesis via deacetylation of regulating proteins, including SIRT1 with $\mathrm{NAD}^{+}$, and suppress HIF1 $\alpha$ and $\mathrm{NF} \kappa \mathrm{B}$ [38-40]. AMPK and $\mathrm{NAD}^{+}$-SIRT1-PGC1 $\beta$ signaling are key factors for nutritional state-dependent M1/M2-like activation of macrophages in inflammatory conditions [39, 41]. HIF- $1 \alpha$ also enhances the lactate dehydrogenase- (LDH-) mediated conversion of pyruvate-to-lactate [42] and increases expression of GLUT1, GLUT3, and MCT4 to increase glucose uptake and expression of pyruvate kinase M2 (PKM2), resulting in an increase in the secretion of lactate and uncoupled glycolysis and oxidative phosphorylation $[43,44]$ (Figure 2). Pyruvate dehydrogenase (PDH) inactivation from phosphorylation by pyruvate dehydrogenase kinases (PDKs) prevents pyruvate from entering the mitochondrial Krebs cycle [45]. HIF- $1 \alpha$ transcriptionally activates the PDKs $[46,47]$.

LPS-activated dendritic cells and M1-like activated macrophages show enhanced aerobic glycolysis, flux through the pentose phosphate pathway, and fatty acid synthesis but have incomplete OXPHOS at the level of succinate dehydrogenase $(\mathrm{SDH})$ and isocitrate dehydrogenase, blocking the synthesis of mitochondrial ATP. In these cells, glucose is used for the biosynthesis of large quantities of cytokines and effector molecules, and inactivation of OXPHOS directs metabolites from the Krebs cycle for inflammatory reaction $[32,48]$. Accumulation of succinate and citrate from the truncated OXPHOS leads to stabilization of HIF1 $\alpha$ by limiting prolyl hydroxylase activity to maintain a proinflammatory, antitumoral response [49-51].

Recently, itaconic acid-mediated inhibition of $\mathrm{SDH}$ has also been found as a driver for succinate accumulation in LPS-stimulated M1-like activated proinflammatory 


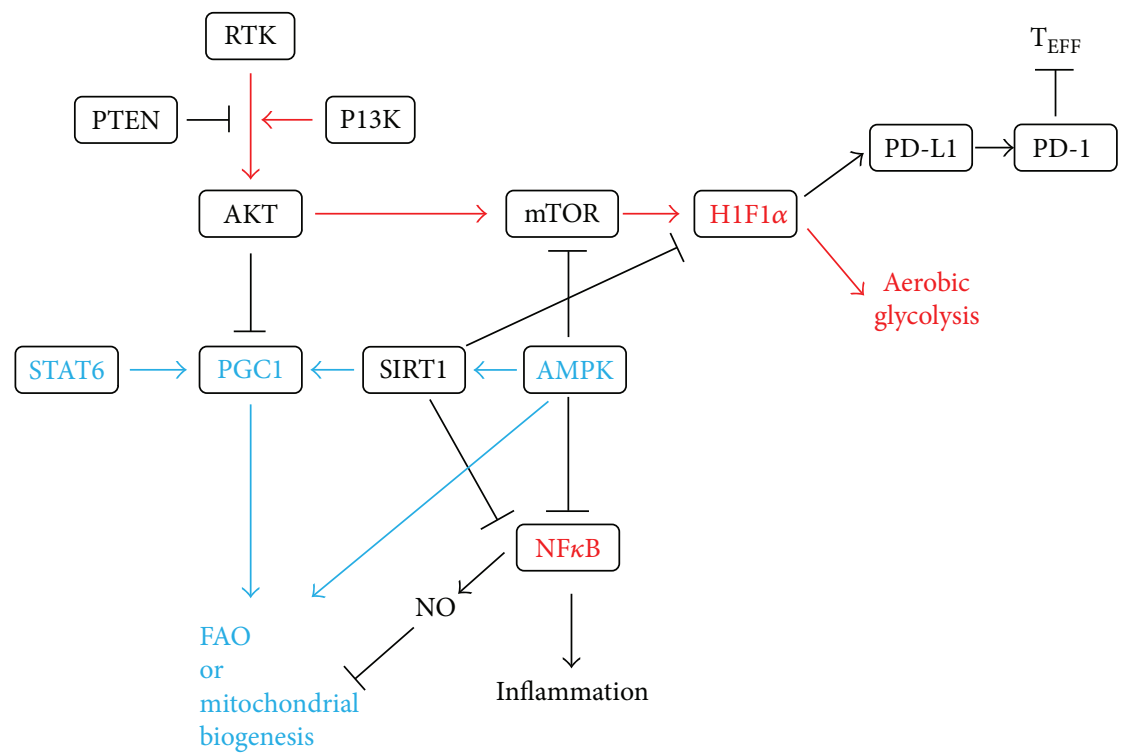

FIGURE 1: Regulation of metabolic rewiring in macrophages. PGC1 is important for FAO and mitochondrial biogenesis (shown in blue) and HIF $1 \alpha$ for aerobic glycolysis (shown in red). Active SIRT1 can inhibit inflammation and glycolytic metabolism and promote mitochondrial biogenesis and FAO. Interaction of PD-L1 and PD-1 induces FAO and suppresses aerobic glycolysis and immune functions in Teff. PD-1: programmed death-1; PD-L1: programmed death ligand-1; RTK: receptor tyrosine kinase; $\mathrm{T}_{\mathrm{EFF}}$ : effector $\mathrm{T}$ cell.

macrophages [52]. Immunoresponsive gene $1(\operatorname{Irg} 1)$ is highly expressed in mammalian macrophages during inflammation and $\operatorname{Irg} 1$ gene silencing in macrophages results in significantly decreased intracellular itaconic acid levels as well as significantly reduced antimicrobial activity during bacterial infections [53].

High intracellular iron levels in M1-like activated macrophages stabilize HIF1 $\alpha$ through low levels of ferroportin and high levels of $\mathrm{H}$-ferritin, involved in iron export and storage, respectively $[54,55]$. Hemeoxygenase-1 (HO-1) catabolizes heme to ferrous ion, biliverdin, and carbon monooxide, and suppression of HO-1 results in M2-like activation of TAMs [56].

HIF $1 \alpha$ can also be stabilized from nitrosylation with peroxynitrites from increased iNOS [57], favoring aerobic glycolysis in $\mathrm{M} 1$ phenotypes. NF $\kappa \mathrm{B}$ transcriptionally activates proinflammatory genes including iNOS, which forms $\mathrm{NO}$ in the presence of arginine. Peroxynitrite, formed from $\mathrm{NO}$ and superoxide anions in the mitochondria, nitrosylates iron-sulfur proteins in the mitochondrial electron transport chain, and the resultant nitrosylation can inhibit OXPHOS [58], also favoring aerobic glycolysis in M1 phenotypes. Unlike iNOS-mediated catabolism of arginine to NO in M1-like activated macrophages, M2-like activated macrophages catalyze arginine to urea and ornithine by arginase 1 (ARG1); ARG1 is a representative marker for M2-like activation. As NO production is limited in M2-like activated macrophages, the nitrosylation-mediated inhibition of OXPHOS is dampened, now favoring M2 phenotypes [48]. Although HIF1 $\alpha$ drives the M1 phenotypes in hypoxic conditions, lactate produced by cancer cells, as a by-product of aerobic glycolysis, has an unexpected critical function in HIF $1 \alpha$ dependent expression of ARG1 and resultant M2-like activation of TAMs in normoxic conditions [59] (Figure 3). These findings clearly indicate highly interconnected signaling for the conservation of HIF-1-centered metabolic phenotypes.

As stated, M2-like activated macrophages show lowered glycolysis and enhanced FAO to fuel OXPHOS. Th2 cytokine and IL-4-induced PGC1 $\beta$ increase mitochondrial biogenesis and FAO in a STAT6-dependent manner $[38,41,60]$. PGC1 $\beta$ plays a key role in increasing mitochondrial biogenesis and OXPHOS by upregulating the expression of FAOinvolved genes [41]. IL-4-/IL-13-stimulated macrophages express PFKFB1, which produces a low level of a glycolytic activator, fructose 2,6 bisphosphate $[61,62]$. In IL-4stimulated macrophages, fatty acid sources such as LDL and VLDL are taken up via the scavenger receptor CD36 and metabolized in the lysosome. The CD36-mediated lysosomal lipolysis is essential for the M2-like activation [31].

An orphan nuclear receptor, estrogen-related receptor $\alpha$ $(\operatorname{ESRR} \alpha)$, is required for the increased mitochondrial biogenesis [63]. Importantly, ESRR $\alpha$-deficient macrophages show a decrease in phagosomal maturation and antimicrobial activity [64]. Another study reported an M1-like phenotype of increased glycolysis but impaired mitochondrial respiratory function and biosynthesis as a result of ESRR $\alpha$ deficiency [65]. Interestingly, VLDLR expression is a determinant factor in inflammation and in M1-like activation of macrophages in AT [66].

In spite of our knowledge gained from macrophages in inflammatory disease conditions, our understanding of the metabolic regulations in TAMs is surprisingly limited and the signals involved in communication between tumors and macrophages are still poorly defined [67]. However, emerging evidence strongly indicates that the metabolic reprogramming of macrophages is closely related to the protumoral or antitumoral function of macrophages $[68,69]$ and that unraveling the TAM phenotype might lead to the 


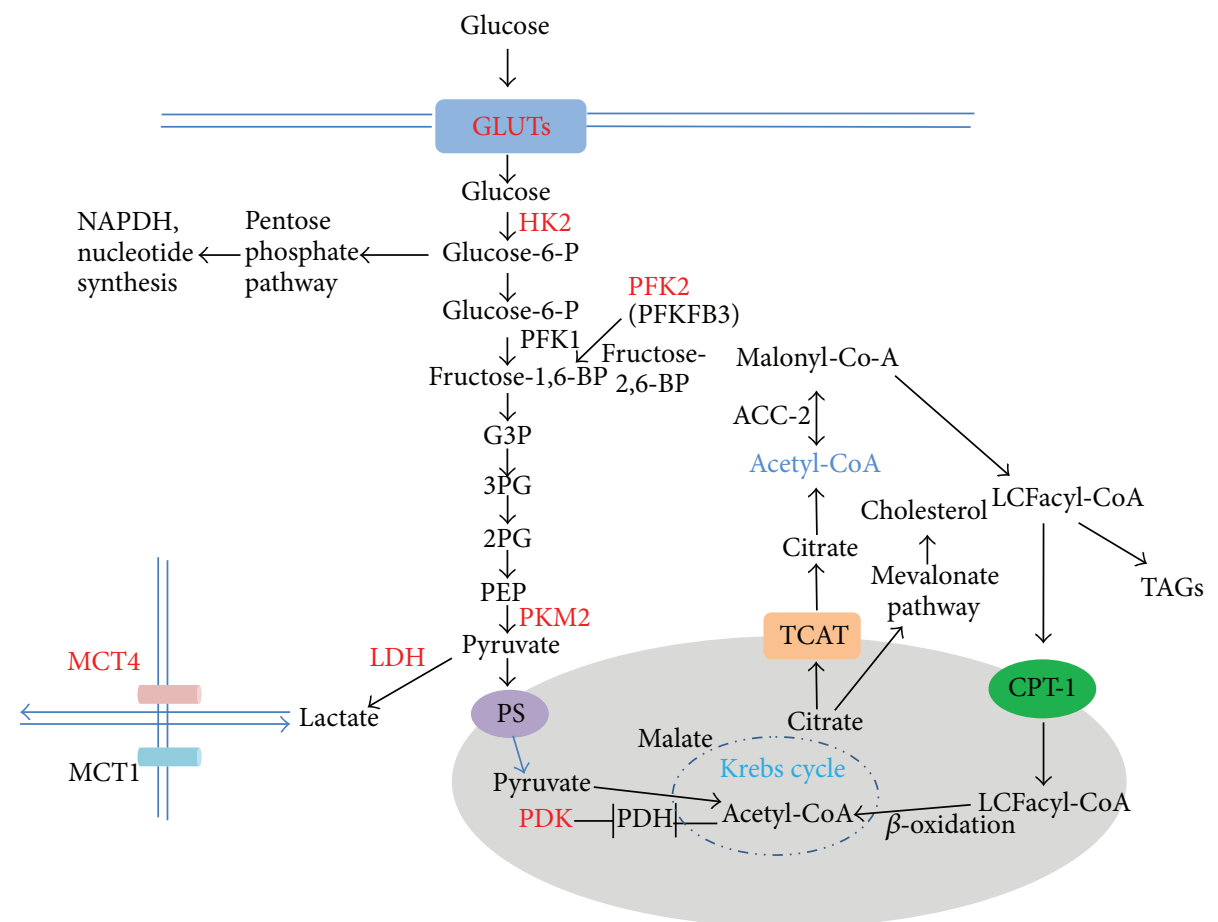

(a)

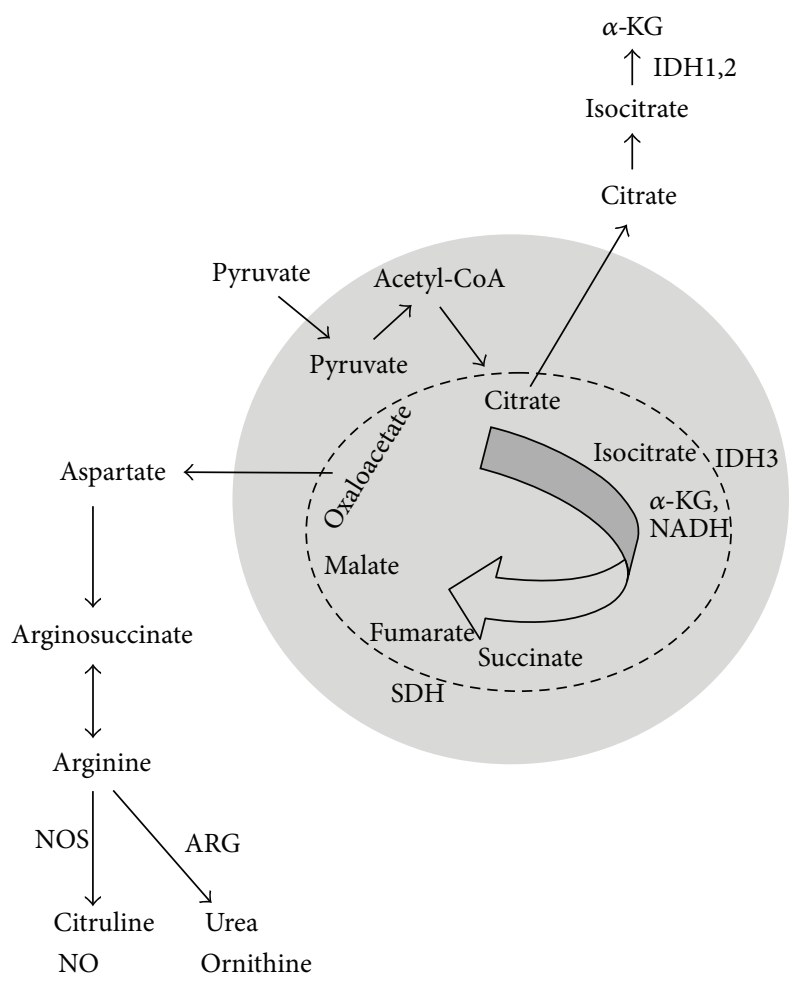

(b)

FIGURE 2: Metabolism of glucose and fatty acid at a glance. (a) Stabilization of HIF-1 $\alpha$ upregulates GLUTs, HK2, PFK2, PKM2, LDH, PDK, and MCT4 shown in red. ACC: acetyl-CoA carboxylase; ARG: arginase; CPT-1: carnitine palmitoyltransferase 1; FAT: fatty acid translocase; G3P: glyceraldehyde 3-phosphate; GLUT: glucose transporter; HK2: hexokinase 2; IDH: isocitrate dehydrogenase; LCFacyl-CoAs: long-chain fatty acyl-CoAs; MCT: monocarboxylate transporter; 2PG: 2-phosphoglycerate; 3PG: 3-phosphoglycerate; PEP: phosphoenolpyruvate; PDH: pyruvate dehydrogenase; PDK: pyruvate dehydrogenase kinase; PFK: phosphofructokinase; PS: pyruvate symporter; SDH: succinate dehydrogenase; TAG: triacylglyceride; TCAT: tricarboxylic acid transporter. (b) A schematic of the Krebs cycle and metabolites exported out of the mitochondria. Arginine is metabolized to urea and ornithine in M2-like macrophages that do not express NOS. ARG: arginase; NOS: nitric oxide synthase. 


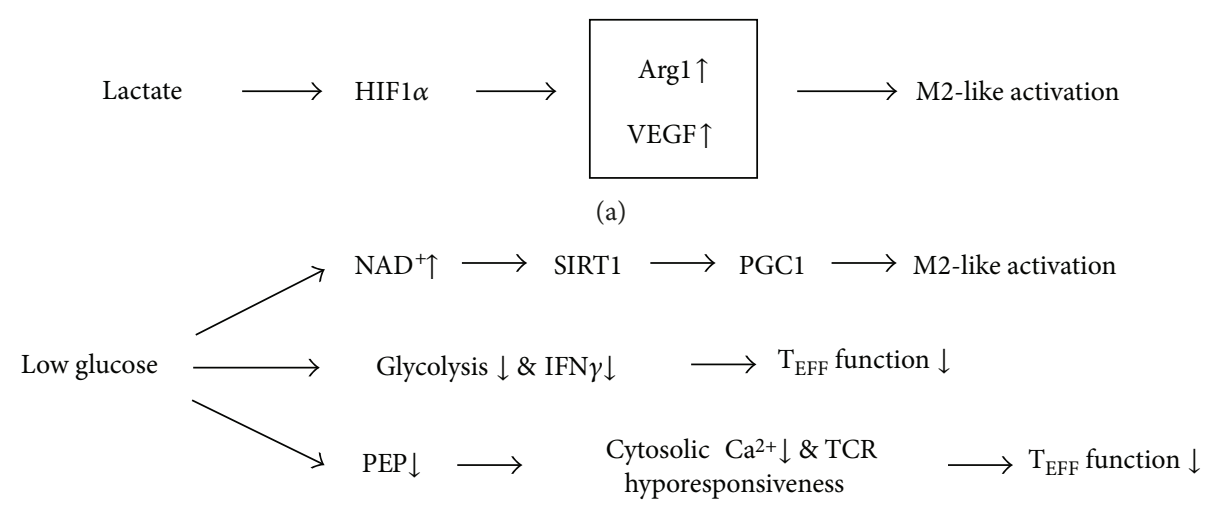

(b)

FIgURE 3: Metabolic changes in the TME-regulating immune cell function. (a) Lactate produced by cancer cells, as a by-product of aerobic glycolysis, has a critical function in inducing M2-like activation of TAMs. (b) A low-glucose microenvironment via multiple signaling pathways regulates activation state of macrophages and T cells. TCR: T cell receptor; VEGF: vascular endothelial growth factor.

identification of alternative, novel metabolic targets for TAM-directed intervention. Recently, it was shown that lactate produced by cancer cells has a critical function in inducing M2-like activation of TAMs [59] (Figure 3). Acidification of the TME by lactate increases level of ARG1, a representative M2 marker, in macrophages, which limits the proinflammatory, antitumoral response of TAMs and, importantly, the proliferation and activation of T cells [59, 70]. Also, de novo fatty acid synthesis in cancer cells increases fatty acid levels in the TME to promote the generation of immunosuppressive, regulatory $\mathrm{T}$ cells (Tregs) and M2-like TAMs, favoring survival of cancer cells [71]. Expression level of vitamin D receptor (VDR) negatively correlates with metastasis in breast cancer, and suppression of VDR by TNF $\alpha$ can mediate the prometastatic effects of TAMs through enhancement of the $\beta$-catenin pathway [72].

\section{Metabolic Regulation of T Cells}

Multiple studies have shown that distinct metabolic programs in $\mathrm{CD}^{+} \mathrm{T}$ cell subsets can be manipulated in vivo to control Treg and effector $\mathrm{T}$ cells $\left(\mathrm{T}_{\mathrm{EFF}}\right)$ development in inflammatory diseases [73-76]. A transcription factor, Myc, shows a dominant role in driving metabolic reprogramming in activated $\mathrm{T}$ cells by promoting glycolysis and glutaminolysis and suppressing FAO [75]. mTOR increases expression of HIF- $1 \alpha$, which facilitates the expression of critical glycolytic enzymes and promotes differentiation and activation of $\mathrm{T}$ cells [76].

A "shift" from OXPHOS to aerobic glycolysis is a hallmark of T cell activation [25]. T cells, if not activated, show low levels of metabolic requirements, use OXPHOS to maximize production of ATP as an energy source, and engage scarcely in biosynthesis, while activated $\mathrm{T}$ cells use aerobic glycolysis to produce effector molecules for rapid cellular proliferation [32].

In order to facilitate proper immunological response upon encounter of antigenic stimuli, it is vital that $\mathrm{T}$ cells should differentiate into $\mathrm{T}_{\mathrm{EFF}}$ and clonally expand rapidly to ensure prompt reaction. Glycolysis promotes the differentiation of activated $\mathrm{CD} 4{ }^{+} \mathrm{T}$ cells into $\mathrm{T}_{\mathrm{EFF}}$ [73]. Activated $\mathrm{T}$ cells also consume glutamine to fuel the Krebs cycle to support the production of biomass and ATP [77]. Clonal expansion is achieved from upregulation of glycolysis and OXPHOS together. In addition to high level of glycolysis, increased mitochondrial flux and production of ROS are also required for initiation of the clonal expansion [78]. After differentiation, $\mathrm{T}_{\mathrm{FFF}}$ cells, Th1, Th2, and Th17 cells, remain highly glycolytic [73].

When the antigenic stimuli are eliminated, most $\mathrm{T}_{\mathrm{EFF}}$ cells die, leaving behind a small antigen-specific $\mathrm{T}$ cell population that becomes memory $\mathrm{T}$ cells (Tm). Quiescent Tm with the CD8 coreceptor exploits FAO to fuel OXPHOS rather than aerobic glycolysis for ATP production [32, 73]. Instead of utilizing extracellular lipids for energy generation, Tm metabolizes de novo generated fatty acids, synthesized from extracellular glucose and intracellularly stored during the previous effector phase [79]. Enforcing FAO with activation of AMPK or inhibiting mTOR results in increased numbers of Tm [80-82]. Mitochondrial oxidative metabolism supports immunosuppression and lineage commitment of Tregs [83-85]. Tregs with increased glycolysis are more proliferative yet have reduced ability to maintain FOXP3 expression and suppress inflammation [84].

\section{Nonmetabolic Function of Glycolytic Enzymes in Immune Cells}

In addition to their canonical, metabolic functions in glycolysis, recent studies uncovered nonmetabolic functions of glycolytic enzymes such as hexokinase 2 (HK2), phosphoglucose isomerase, and GAPDH, connecting metabolic states to apoptosis, gene transcription, protein kinase activity, and the mTOR signaling pathway [86]. Briefly, the interaction between HK2 and voltage-dependent anion channel (VDAC1) reduces the release of proapoptotic proteins and prevents cancer cells from undergoing apoptosis [87]; phosphoglucose isomerase exerts its antiapoptosis effect by suppressing the expression of Apaf- 1 and caspase- 9 genes, thereby indirectly regulating the formation of the 
apoptosome [88, 89]. GAPDH exerts controversial pro- and antiapoptotic effects through interaction with VDAC1 and induction of autophagy, respectively $[86,90]$.

Other studies have shown that GAPDH, HK, and enolase are also RNA-binding proteins [91-93] and the "REM (RNA-enzyme-metabolite) hypothesis" proposes a regulatory interaction between gene expression and cellular metabolism by RNA-binding metabolic enzymes [94, 95]. It is notable that many glycolytic enzymes, formerly known to exclusively function in glycolytic metabolic events in the cytoplasm or mitochondria, have now been shown to regulate transcription and translation [86, 91]. Indeed, numerous metabolic enzymes that also function in glycolysis, fatty acid synthesis, and the Krebs cycle are also RNA-binding proteins [96], although the significance for immune response is not clearly known except for that of GAPDH [95] and enolase $[32,83]$. Recently, a REM connection with GAPDH was proven in T cell activation [25]. GAPDH is diverted to glycolysis and translation of IFN $\gamma$ and IL-2 is not perturbed in highly glycolytic $\mathrm{T}$ cells. However, when aerobic glycolysis is blocked, GAPDH binds to IFN $\gamma$ and IL-2 mRNA in $\mathrm{CD}^{+} \mathrm{T}$ cells to suppress their translation [25]. In myeloid cells, GAPDH is a component of the IFN $\gamma$-activated inhibitor of translation (GAIT) complex that controls translation of inflammatory genes $[97,98]$. High glycolytic flux suppresses the interaction between GAPDH and Rheb and thus allows Rheb to activate mTORC1 and stimulate cell growth [99]. By modulating expression of Foxp3-splicing variants with exon 2(Foxp3-E2), enolase-1-mediated glycolysis controls induction of human Tregs with a potent immunosuppressive function [83]. When glycolysis is inhibited, enolase- 1 translocates to the nucleus and represses expression of the Foxp3-E2 splice variant in Tregs and suppresses Treg induction.

The level of the glycolytic intermediate phosphoenolpyruvate (PEP) is controlled by a balance between enolasemediated formation of PEP and pyruvate kinase-mediated conversion to pyruvate. PKM2 exists either as an inactive dimer or as more active tetramer, and the transition between the two conformations is subject to posttranslational modifications [100]. Dimeric PKM2, previously regarded as crucial for metabolic reprogramming exclusively in cancer cell, is also important in promoting aerobic glycolysis in immune cells $[101,102]$. Enhanced expression of dimeric PKM2 reduces the rate of PEP conversion to pyruvate and results in an accumulation of glycolytic products that can be otherwise metabolized in biosynthetic pathways [32]. Importantly, PEP enhances antitumor effector functions in activated $\mathrm{T}$ cells by regulating $\mathrm{Ca}_{2}^{+}$import into the endoplasmic reticulum, thus sustaining translocation of nuclear factor of activated $\mathrm{T}$ cells (NFAT) into the nucleus and the expression of a set of genes that are required for $\mathrm{T}$ cell activation [103].

These findings imply that a direct and strong interaction exists between the nonmetabolic function of glycolytic enzymes and the generation of immune responses and also that enhanced glycolysis sustains antitumoral and proinflammatory functions via highly interconnected signaling in immune cells.

\section{Metabolic Changes in the TME Influencing Immune Cell Functions}

The microenvironment determines the metabolism of immune cells, which in turn adjust to a broad spectrum of configurations to meet the demands of various cellular activities. For example, changes in the metabolic profiles of immune cells by cancer cells can alter the function of the immune cells [103, 104]. A protracted aerobic glycolysis acidifies and destabilizes the TME and this is consistent with the view of the tumor as an unhealed wound [105]. Similarities of utilizing nutrients and engaging metabolic regulation to sustain cellular proliferation and survival are shared by cancer and immune cells. Notably, nutritional competition between cancer cells and antitumoral immune cells in the TME shifts the activation and differentiation status of T-cells to favoring the survival of cancer cells $[103,104,106,107]$.

Recently, it was shown that lactate produced by cancer cells, as a by-product of aerobic glycolysis, has a critical function in signaling that induces M2-like activation of TAMs [59] (Figure 3). Interestingly, lactate-induced M2-like activation was from HIF1 $\alpha$-dependent expression of ARG1. Depletion of glucose and a glucose-rich hypoxic ROS environment favor M2-like activation and M1-like activation of TAMs, respectively, and depletion of glucose can disarm $\mathrm{T}$ cells in the TME [27, 104]. Low levels of ATP from dietary restrictions or energy consumption induces nicotinamide phosphoribosyltransferase that generates $\mathrm{NAD}^{+}$, which is a key factor for SIRT1 activation. SIRT1 acetylates and activates PGC1 $\beta$ to increase OXPHOS $[35,67,108]$. Pyruvate is metabolized by $\mathrm{LDH}-\mathrm{A}$, producing lactate and $\mathrm{NAD}^{+}$. $\mathrm{NAD}^{+}$acts as an electron acceptor in the Krebs cycle and the electron transport system in mitochondria. It appears feasible from these findings that glucose-depleted, low ATP, and $\mathrm{NAD}^{+}$-rich states (in cachexic patient with advanced cancer) may drive the M2-like activation of macrophages, while the macrophage population still retains its phagocytic activity in maintaining biosynthesis with molecules acquired from their microenvironment [32]. For the identification of alternative, novel targets for TAM-directed intervention, it would be necessary to show whether these events can predominantly happen in TAMs of the TME.

A recent study observed that hypoxia-induced upregulation of the immunosuppressive programmed death ligand1 (PD-L1) is directly mediated by HIF $1 \alpha$ [109]. In the TME, cancer cells, macrophages, and dendritic cells express PDL1, a notable ligand for immune checkpoint, programmed cell death-1(PD-1) in $\mathrm{T}_{\mathrm{EFF}}$. The interaction of $\mathrm{PD}-1$ and PD-L1 directly inhibits glycolysis and promotes lipolysis and FAO in T cells, resulting in failure of the antitumoral function of T cells [110] (Figure 1). The lessons that application of immune checkpoint blockade antibodies against cytotoxic T lymphocyte antigen-4 (CTLA-4), PD-1, and PD-L1, which are used clinically, restore glucose in the TME, permitting $\mathrm{T}$ cell glycolysis and IFN $\gamma$ production clearly show that nutrient availability in the microenvironment can change the metabolic status of immune cells. Another study also 
revealed that PD-1 expression by TAMs correlates with protumoral activity, and blockage of PD-1-PD-L1 in vivo increases phagocytosis, reduces growth of cancer cells, and increases the survival of mice in mouse models of cancer in a macrophage-dependent way [111]. In addition, blocking PD-L1 directly on cancer cells decreases glycolysis and restores glucose in the TME, resulting in allowance of antitumoral function of $\mathrm{T}$ cells from glycolysis and IFN $\gamma$ production [104].

In conclusion, these findings indicate that signals such as cytokines, growth factors, hypoxia, and nutrient availability that emanate from the microenvironment can induce metabolic changes in immune cell subsets, resulting in changes in immune functions and pathological responses. An interesting perspective is whether immune cells can supply their microenvironment with lactate and antioxidative resources as stromal cells in the TME are able to (the reverse Warburg effect). Considering their preponderance in the TME, there is an ample possibility that metabolic changes in a large subgroup of macrophages or $\mathrm{T}$ cells may also affect the metabolic state of their microenvironment and functions of other cellular components.

\section{Potential Metabolic Targets for the Manipulation of Immune Cell Function}

Since the function of immune cells is dependent on a delicate metabolic balance, results of many clinical trials performed with inhibitors of metabolic enzymes and oncogenes will provide valuable insights for the prospect of immunomodulation by specific metabolic regulation [112]. Of note, results from targeting cancer metabolism in vivo have been disappointing and less prominent than results from targeting immune cell metabolism [85]. The PKM2 inhibitor, TLN232, was tested in a clinical trial for refractory renal cell carcinoma (NCT00422786). Inactive dimeric PKM2 activates the mTORC1 signaling pathway by phosphorylating the mTOR inhibitor, AKT1S1, and leads to an accelerated oncogenic growth and autophagy inhibition of cancer cells [113]. In line with this, increase in the tetrameric, active form of PKM2, attenuated the LPS-induced proinflammatory M1like macrophage phenotypes while promoting M2-like macrophage phenotypes [114]. Many AMPK activators are now tested in clinical and preclinical studies for diabetes, cancer, and cardiovascular disease [115]. Importantly, AMPK stimulation inhibiting mTORC1 was sufficient to decrease Glut1 and increase generation of Tregs in an animal model, implying AMPK activation as a potential manipulable checkpoint for immune response [73]. REDD1, an inhibitor of mTOR, is highly expressed in M2-like TAMs. Inhibition of REDD1 stimulates glycolysis in the TAMs and competition of glucose between TAMs and endothelial cells prevents vascular hyperactivation and promotes the formation of quiescent vascular junctions in the TME [69]. Suppression of REDD1 was attempted in phase 2 clinical trial (NCT00713518) for the treatment of neovascularization in AMD patients. Nitrosylation of HIF $1 \alpha$ prevents its degradation. If denitrosylation of HIF $1 \alpha$ is observed, its modulation may be potentially applicable for the inhibition of glycolytic enzymes and the alleviation of M1-like phenotypes.

Isoprenylation of ubiquinone is important for OXPHOS and isoprenylation of Ras, Rho, and Rab guanosine triphosphatases is involved in immunological synapse formation, migration, proliferation, and cytotoxic effector response of $\mathrm{T}$ cells. The intracellular availability of sterols is crucial for isoprenylation modification of proteins for plasma membrane attachment and represents a checkpoint for metabolic reprogramming that modulates $\mathrm{T}$ cell responses [116]. Statin and other chemical inhibitors of the mevalonate pathway can suppress isoprenylation of Rho proteins [117] and have been tested in many clinical trials.

Clinical trials involving agents that inhibit PD-L1 and PD-1 are now being performed. Atezolizumab is the sole member of this class currently approved for the treatment of bladder cancer, but approvals for avelumab, durvalumab, nivolumab, and pembrolizumab in the treatment of various cancer are anticipated in the near future [118]. Therefore, it appears possible that the combined use of metabolismtargeting reagents with immune checkpoint inhibitors can alter the activation and differentiation of $\mathrm{T}$ cells.

\section{Conclusions}

Immunity and metabolism advance together. Considering the significant contribution of immune cell functions in promoting and suppressing various types of disease progression, repolarization of immune cells from the potential targets stated above shows an ample possibility to become novel therapeutic approaches. Extension of our knowledge of the functional plasticity of macrophages and $\mathrm{T}$ cells spanning from inflammation biology to cancer immunology and the persistent reprogramming effect achievable from stable epigenetic changes in the metabolic pathways of macrophages [29] and potentially $\mathrm{T}$ cells by potential modulators may provide new information for immune therapeutic strategies applicable for different disease conditions. Importantly, cancer cells and host primary cell constituents such as immune cells and stromal cells can form microanatomical compartments within the cancer tissue to regulate metabolic needs, immune surveillance, survival, invasion, and metastasis. Indeed, different signals from particular locations in the TME seem to influence activation of TAMs and T cells and overall tumor prognosis [119]. TAMs can be diverse within the microanatomical compartments, including the accumulation of M1-like activated cells with protumoral properties in hypoxic areas [120] and differences in inflammatory components and pathways between tumors originating in distinct anatomical sites $[120,121]$. The notion that metabolic competition between cancer cells, immune cells, and other stromal cells can determine function and fate of each cell subset proposing that identification of which of specific niches in the microenvironment can impede immune cells from proper metabolic engagement will encourage significant contributions to this research field. Generation of metabolically fit $\mathrm{T}$ cells prior to adoptive cell transfer will improve $\mathrm{T}$ cell-based immunotherapy against cancer by surviving the unfavorable, hostile TME. Furthermore, successful therapies 
targeting the function of macrophages and T cells will require identification of targets that specifically allow metabolic reprogramming of immune cells while, at the same time, not causing an increase in proliferation and survival of cancer cells or systemic inflammatory changes or autoimmunity. Our understanding of the metabolic regulations in B cells is surprisingly limited, and the mechanisms about how cellular metabolism supports and regulates function of B cells are still poorly defined. B cell immunometabolism is anticipated to become an exciting research field.

\section{Conflicts of Interest}

The authors declare that they have no conflicts of interest.

\section{Acknowledgments}

This study was supported by a grant of the Korea Institute of Radiological and Medical Sciences (KIRAMS), funded by Ministry of Science and ICT (MSIT), Republic of Korea (1711045557, 1711045538, and 1711045554) and by the Basic Science Research Program through the National Research Foundation of Korea (NRF of Korea) funded by the Ministry of Science, ICT \& Future Planning (NRF-2017R1D1A1B03029063).

\section{References}

[1] H. F. Dvorak, "Tumors: wounds that do not heal. Similarities between tumor stroma generation and wound healing," The New England Journal of Medicine, vol. 315, no. 26, pp. 1650-1659, 1986.

[2] L. Bingle, N. J. Brown, and C. E. Lewis, "The role of tumourassociated macrophages in tumour progression: implications for new anticancer therapies," The Journal of Pathology, vol. 196, no. 3, pp. 254-265, 2002.

[3] J. M. Fernandez-Real and J. C. Pickup, "Innate immunity, insulin resistance and type 2 diabetes," Trends in Endocrinology and Metabolism, vol. 19, no. 1, pp. 10-16, 2008.

[4] D. A. Winer, S. Winer, L. Shen et al., "B cells promote insulin resistance through modulation of $\mathrm{T}$ cells and production of pathogenic IgG antibodies," Nature Medicine, vol. 17, no. 5, pp. 610-617, 2011.

[5] J. Jager, M. Aparicio-Vergara, and M. Aouadi, "Liver innate immune cells and insulin resistance: the multiple facets of Kupffer cells," Journal of Internal Medicine, vol. 280, no. 2, pp. 209-220, 2016.

[6] P. M. Kelly, R. S. Davison, E. Bliss, and J. O. McGee, "Macrophages in human breast disease: a quantitative immunohistochemical study," British Journal of Cancer, vol. 57, no. 2, pp. 174-177, 1988.

[7] E. Van Overmeire, D. Laoui, J. Keirsse, J. A. Van Ginderachter, and A. Sarukhan, "Mechanisms driving macrophage diversity and specialization in distinct tumor microenvironments and parallelisms with other tissues," Frontiers in Immunology, vol. 5, p. 127, 2014.

[8] A. Mantovani, A. Sica, S. Sozzani, P. Allavena, A. Vecchi, and M. Locati, "The chemokine system in diverse forms of macrophage activation and polarization," Trends in Immunology, vol. 25 , no. 12, pp. 677-686, 2004.
[9] Y. C. Wang, F. He, F. Feng et al., "Notch signaling determines the M1 versus M2 polarization of macrophages in antitumor immune responses," Cancer Research, vol. 70, no. 12, pp. 4840-4849, 2010.

[10] F. Rae, K. Woods, T. Sasmono et al., "Characterisation and trophic functions of murine embryonic macrophages based upon the use of a Csf1r-EGFP transgene reporter," Developmental Biology, vol. 308, no. 1, pp. 232-246, 2007.

[11] A. Sica and A. Mantovani, "Macrophage plasticity and polarization: in vivo veritas," The Journal of Clinical Investigation, vol. 122, no. 3, pp. 787-795, 2012.

[12] P. J. Murray, J. E. Allen, S. K. Biswas et al., "Macrophage activation and polarization: nomenclature and experimental guidelines," Immunity, vol. 41, no. 1, pp. 14-20, 2014.

[13] J. Van den Bossche, J. Baardman, and M. P. de Winther, "Metabolic characterization of polarized M1 and M2 bone marrow-derived macrophages using real-time extracellular flux analysis," Journal of Visualized Experiments, no. 105, article e53424, 2015.

[14] B. Z. Qian and J. W. Pollard, "Macrophage diversity enhances tumor progression and metastasis," Cell, vol. 141, no. 1, pp. 39-51, 2010.

[15] Q. W. Zhang, L. Liu, C. Y. Gong et al., "Prognostic significance of tumor-associated macrophages in solid tumor: a meta-analysis of the literature," PLoS One, vol. 7, no. 12, article e50946, 2012.

[16] A. Mantovani, P. Allavena, A. Sica, and F. Balkwill, "Cancer-related inflammation," Nature, vol. 454, no. 7203, pp. 436-444, 2008.

[17] M. Zhang, Y. He, X. Sun et al., "A high M1/M2 ratio of tumor-associated macrophages is associated with extended survival in ovarian cancer patients," Journal of Ovarian Research, vol. 7, no. 1, p. 19, 2014.

[18] A. Yuan, Y. J. Hsiao, H. Y. Chen et al., "Opposite effects of M1 and M2 macrophage subtypes on lung cancer progression," Scientific Reports, vol. 5, no. 1, p. 14273, 2015.

[19] S. P. Weisberg, D. McCann, M. Desai, M. Rosenbaum, R. L. Leibel, and A. W. Ferrante Jr, "Obesity is associated with macrophage accumulation in adipose tissue," The Journal of Clinical Investigation, vol. 112, no. 12, pp. 1796-1808, 2003.

[20] C. N. Lumeng, J. L. Bodzin, and A. R. Saltiel, “Obesity induces a phenotypic switch in adipose tissue macrophage polarization," The Journal of Clinical Investigation, vol. 117, no. 1, pp. 175-184, 2007.

[21] A. Castoldi, C. Naffah de Souza, N. O. Camara, and P. M. Moraes-Vieira, "The macrophage switch in obesity development," Frontiers in Immunology, vol. 6, p. 637, 2016.

[22] S. Nishimura, I. Manabe, M. Nagasaki et al., "CD8 ${ }^{+}$effector T cells contribute to macrophage recruitment and adipose tissue inflammation in obesity," Nature Medicine, vol. 15, no. 8, pp. 914-920, 2009.

[23] O. Warburg, F. Wind, and E. Negelein, "The metabolism of tumors in the body," The Journal of General Physiology, vol. 8, no. 6, pp. 519-530, 1927.

[24] M. Chang, J. A. Hamilton, G. M. Scholz, and C. L. Elsegood, "Glycolytic control of adjuvant-induced macrophage survival: role of PI3K, MEK1/2, and Bcl-2," Journal of Leukocyte Biology, vol. 85, no. 6, pp. 947-956, 2009.

[25] C. H. Chang, J. D. Curtis, L. B. Maggi Jr et al., "Posttranscriptional control of $\mathrm{T}$ cell effector function by aerobic glycolysis," Cell, vol. 153, no. 6, pp. 1239-1251, 2013. 
[26] S. K. Biswas, "Metabolic reprogramming of immune cells in cancer progression," Immunity, vol. 43, no. 3, pp. 435-449, 2015.

[27] M. Corrado, L. Scorrano, and S. Campello, "Changing perspective on oncometabolites: from metabolic signature of cancer to tumorigenic and immunosuppressive agents," Oncotarget, vol. 7, no. 29, pp. 46692-46706, 2016.

[28] M. G. Netea, J. Quintin, and J. W. van der Meer, "Trained immunity: a memory for innate host defense," Cell Host \& Microbe, vol. 9, no. 5, pp. 355-361, 2011.

[29] S. C. Cheng, J. Quintin, R. A. Cramer et al., "mTOR- and HIF- $1 \alpha$-mediated aerobic glycolysis as metabolic basis for trained immunity," Science, vol. 345, no. 6204, article 1250684, 2014.

[30] D. Vats, L. Mukundan, J. I. Odegaard et al., "Oxidative metabolism and PGC- $1 \beta$ attenuate macrophage-mediated inflammation," Cell Metabolism, vol. 4, no. 1, pp. 13-24, 2006.

[31] S. C. Huang, B. Everts, Y. Ivanova et al., "Cell-intrinsic lysosomal lipolysis is essential for alternative activation of macrophages," Nature Immunology, vol. 15, no. 9, pp. 846855, 2014.

[32] N. Assmann and D. K. Finlay, "Metabolic regulation of immune responses: therapeutic opportunities," The Journal of Clinical Investigation, vol. 126, no. 6, pp. 2031-2039, 2016.

[33] T. Cramer, Y. Yamanishi, B. E. Clausen et al., "HIF-1alpha is essential for myeloid cell-mediated inflammation," Cell, vol. 112, no. 5, pp. 645-657, 2003.

[34] C. H. Fong, M. Bebien, A. Didierlaurent et al., "An antiinflammatory role for IKK $\beta$ through the inhibition of "classical" macrophage activation," The Journal of Experimental Medicine, vol. 205, no. 6, pp. 1269-1276, 2008.

[35] A. Chawla, "Control of macrophage activation and function by PPARs," Circulation Research, vol. 106, no. 10, pp. 15591569, 2010.

[36] N. Wang, H. Liang, and K. Zen, "Molecular mechanisms that influence the macrophage m1-m2 polarization balance," Frontiers in Immunology, vol. 5, p. 614, 2014.

[37] N. Kapoor, J. Niu, Y. Saad et al., "Transcription factors STAT6 and KLF4 implement macrophage polarization via the dual catalytic powers of MCPIP," Journal of Immunology, vol. 194, no. 12, pp. 6011-6023, 2015.

[38] H. R. Griffiths, D. Gao, and C. Pararasa, "Redox regulation in metabolic programming and inflammation," Redox Biology, vol. 12, pp. 50-57, 2017.

[39] C. Canto, Z. Gerhart-Hines, J. N. Feige et al., "AMPK regulates energy expenditure by modulating $\mathrm{NAD}^{+}$metabolism and SIRT1 activity," Nature, vol. 458, no. 7241, pp. 1056-1060, 2009.

[40] D. B. Shackelford, D. S. Vasquez, J. Corbeil et al., "mTOR and HIF- $1 \alpha$-mediated tumor metabolism in an LKB1 mouse model of Peutz-Jeghers syndrome," Proceedings of the National Academy of Sciences of the United States of America, vol. 106, no. 27, pp. 11137-11142, 2009.

[41] L. A. O'Neill and D. G. Hardie, "Metabolism of inflammation limited by AMPK and pseudo-starvation," Nature, vol. 493, no. 7432, pp. 346-355, 2013.

[42] J. G. Sonanez-Organis, M. Rodriguez-Armenta, B. LealRubio, A. B. Peregrino-Uriarte, S. Gomez-Jimenez, and G. Yepiz-Plascencia, "Alternative splicing generates two lactate dehydrogenase subunits differentially expressed during hypoxia via HIF-1 in the shrimp Litopenaeus vannamei," Biochimie, vol. 94, no. 5, pp. 1250-1260, 2012.

[43] M. S. Ullah, A. J. Davies, and A. P. Halestrap, "The plasma membrane lactate transporter MCT4, but not MCT1, is upregulated by hypoxia through a HIF- $1 \alpha$-dependent mechanism," The Journal of Biological Chemistry, vol. 281, no. 14, pp. 9030-9037, 2006.

[44] H. Pelicano, W. Lu, Y. Zhou et al., "Mitochondrial dysfunction and reactive oxygen species imbalance promote breast cancer cell motility through a CXCL14-mediated mechanism," Cancer Research, vol. 69, no. 6, pp. 2375-2383, 2009.

[45] T. Zhao, Y. Zhu, A. Morinibu et al., "HIF-1-mediated metabolic reprogramming reduces ROS levels and facilitates the metastatic colonization of cancers in lungs," Scientific Reports, vol. 4, p. 3793, 2014.

[46] J. W. Kim, I. Tchernyshyov, G. L. Semenza, and C. V. Dang, "HIF-1-mediated expression of pyruvate dehydrogenase kinase: a metabolic switch required for cellular adaptation to hypoxia," Cell Metabolism, vol. 3, no. 3, pp. 177-185, 2006.

[47] C. W. Lu, S. C. Lin, K. F. Chen, Y. Y. Lai, and S. J. Tsai, "Induction of pyruvate dehydrogenase kinase-3 by hypoxiainducible factor- 1 promotes metabolic switch and drug resistance," The Journal of Biological Chemistry, vol. 283, no. 42, pp. 28106-28114, 2008.

[48] J. Van den Bossche, J. Baardman, N. A. Otto et al., "Mitochondrial dysfunction prevents repolarization of inflammatory macrophages," Cell Reports, vol. 17, no. 3, pp. 684-696, 2016.

[49] G. M. Tannahill, A. M. Curtis, J. Adamik et al., "Succinate is an inflammatory signal that induces IL- $1 \beta$ through HIF- $1 \alpha$," Nature, vol. 496, no. 7444, pp. 238-242, 2013.

[50] K. Ito and T. Suda, "Metabolic requirements for the maintenance of self-renewing stem cells," Nature Reviews. Molecular Cell Biology, vol. 15, no. 4, pp. 243-256, 2014.

[51] P. Koivunen, M. Hirsila, A. M. Remes, I. E. Hassinen, K. I. Kivirikko, and J. Myllyharju, "Inhibition of hypoxiainducible factor (HIF) hydroxylases by citric acid cycle intermediates: possible links between cell metabolism and stabilization of HIF," The Journal of Biological Chemistry, vol. 282, no. 7, pp. 4524-4532, 2007.

[52] V. Lampropoulou, A. Sergushichev, M. Bambouskova et al., "Itaconate links inhibition of succinate dehydrogenase with macrophage metabolic remodeling and regulation of inflammation," Cell Metabolism, vol. 24, no. 1, pp. 158-166, 2016.

[53] A. Michelucci, T. Cordes, J. Ghelfi et al., "Immune-responsive gene 1 protein links metabolism to immunity by catalyzing itaconic acid production," Proceedings of the National Academy of Sciences of the United States of America, vol. 110, no. 19, pp. 7820-7825, 2013.

[54] G. Cairo, S. Recalcati, A. Mantovani, and M. Locati, "Iron trafficking and metabolism in macrophages: contribution to the polarized phenotype," Trends in Immunology, vol. 32, no. 6, pp. 241-247, 2011.

[55] I. Siegert, J. Schodel, M. Nairz et al., "Ferritin-mediated iron sequestration stabilizes hypoxia-inducible factor- $1 \alpha$ upon LPS activation in the presence of ample oxygen," Cell Reports, vol. 13, no. 10, pp. 2048-2055, 2015.

[56] R. Deng, S. M. Wang, T. Yin et al., "Inhibition of tumor growth and alteration of associated macrophage cell type 
by an HO-1 inhibitor in breast carcinoma-bearing mice," Oncology Research, vol. 20, no. 10, pp. 473-482, 2013.

[57] F. Li, P. Sonveaux, Z. N. Rabbani et al., "Regulation of HIFlalpha stability through S-nitrosylation," Molecular Cell, vol. 26, no. 1, pp. 63-74, 2007.

[58] R. J. Mailloux and W. G. Willmore, "S-glutathionylation reactions in mitochondrial function and disease," Frontiers in Cell and Development Biology, vol. 2, p. 68, 2014.

[59] O. R. Colegio, N. Q. Chu, A. L. Szabo et al., "Functional polarization of tumour-associated macrophages by tumourderived lactic acid," Nature, vol. 513, no. 7519, pp. 559-563, 2014.

[60] S. J. Bensinger and P. Tontonoz, "Integration of metabolism and inflammation by lipid-activated nuclear receptors," Nature, vol. 454, no. 7203, pp. 470-477, 2008.

[61] J. C. Rodriguez-Prados, P. G. Traves, J. Cuenca et al., "Substrate fate in activated macrophages: a comparison between innate, classic, and alternative activation," Journal of Immunology, vol. 185, no. 1, pp. 605-614, 2010.

[62] X. Geeraerts, E. Bolli, S. M. Fendt, and J. A. Van Ginderachter, "Macrophage metabolism as therapeutic target for cancer, atherosclerosis, and obesity," Frontiers in Immunology, vol. 8, p. 289, 2017.

[63] S. N. Schreiber, R. Emter, M. B. Hock et al., "The estrogen-related receptor alpha $(\mathrm{ERR} \alpha)$ functions in PPAR $\gamma$ coactivator $1 \alpha$ (PGC- $1 \alpha)$-induced mitochondrial biogenesis," Proceedings of the National Academy of Sciences of the United States of America, vol. 101, no. 17, pp. 64726477, 2004.

[64] S. Y. Kim, C. S. Yang, H. M. Lee et al., "ESRRA (estrogenrelated receptor $\alpha$ ) is a key coordinator of transcriptional and post-translational activation of autophagy to promote innate host defense," Autophagy, pp. 1-17, 2017.

[65] J. M. Yuk, T. S. Kim, S. Y. Kim et al., "Orphan nuclear receptor $\mathrm{ERR} \alpha$ controls macrophage metabolic signaling and A20 expression to negatively regulate TLR-induced inflammation," Immunity, vol. 43, no. 1, pp. 80-91, 2015.

[66] A. Nguyen, H. Tao, M. Metrione, and T. Hajri, "Very low density lipoprotein receptor (VLDLR) expression is a determinant factor in adipose tissue inflammation and adipocytemacrophage interaction," The Journal of Biological Chemistry, vol. 289, no. 3, pp. 1688-1703, 2014.

[67] J. Kim and J. S. Bae, "Metabolic regulation of macrophages in tumor microenvironment," Current Opinion in Hematology, vol. 25, no. 1, pp. 52-59, 2017.

[68] H. L. Penny, J. L. Sieow, G. Adriani et al., "Warburg metabolism in tumor-conditioned macrophages promotes metastasis in human pancreatic ductal adenocarcinoma," OncoImmunology, vol. 5, no. 8, article e1191731, 2016.

[69] M. Wenes, M. Shang, M. Di Matteo et al., "Macrophage metabolism controls tumor blood vessel morphogenesis and metastasis," Cell Metabolism, vol. 24, no. 5, pp. 701-715, 2016.

[70] T. Ohashi, T. Akazawa, M. Aoki et al., "Dichloroacetate improves immune dysfunction caused by tumor-secreted lactic acid and increases antitumor immunoreactivity," International Journal of Cancer, vol. 133, no. 5, pp. 1107-1118, 2013.

[71] L. Zhu, Q. Zhao, T. Yang, W. Ding, and Y. Zhao, "Cellular metabolism and macrophage functional polarization,"
International Reviews of Immunology, vol. 34, no. 1, pp. 82100, 2015.

[72] Y. Zhang, Q. Guo, Z. Zhang et al., "VDR status arbitrates the prometastatic effects of tumor-associated macrophages," Molecular Cancer Research, vol. 12, no. 8, pp. 1181-1191, 2014.

[73] R. D. Michalek, V. A. Gerriets, S. R. Jacobs et al., "Cutting edge: distinct glycolytic and lipid oxidative metabolic programs are essential for effector and regulatory $\mathrm{CD} 4^{+} \mathrm{T}$ cell subsets," Journal of Immunology, vol. 186, no. 6, pp. 32993303, 2011.

[74] A. N. Macintyre, V. A. Gerriets, A. G. Nichols et al., "The glucose transporter Glut1 is selectively essential for CD4 T cell activation and effector function," Cell Metabolism, vol. 20, no. 1, pp. 61-72, 2014.

[75] R. Wang, C. P. Dillon, L. Z. Shi et al., "The transcription factor Myc controls metabolic reprogramming upon T lymphocyte activation," Immunity, vol. 35 , no. 6, pp. 871-882, 2011.

[76] E. V. Dang, J. Barbi, H. Y. Yang et al., "Control of $\mathrm{T}_{\mathrm{H}} 17 / \mathrm{T}_{\mathrm{reg}}$ balance by hypoxia-inducible factor 1," Cell, vol. 146, no. 5, pp. 772-784, 2011.

[77] E. L. Carr, A. Kelman, G. S. Wu et al., "Glutamine uptake and metabolism are coordinately regulated by ERK/MAPK during T lymphocyte activation," Journal of Immunology, vol. 185, no. 2, pp. 1037-1044, 2010.

[78] L. A. Sena, S. Li, A. Jairaman et al., "Mitochondria are required for antigen-specific $\mathrm{T}$ cell activation through reactive oxygen species signaling," Immunity, vol. 38 , no. 2, pp. 225-236, 2013.

[79] D. O'Sullivan, G. J. van der Windt, S. C. Huang et al., "Memory $\mathrm{CD}^{+} \mathrm{T}$ cells use cell-intrinsic lipolysis to support the metabolic programming necessary for development," Immunity, vol. 41, no. 1, pp. 75-88, 2014.

[80] E. L. Pearce, M. C. Walsh, P. J. Cejas et al., "Enhancing CD8 T-cell memory by modulating fatty acid metabolism," Nature, vol. 460, no. 7251, pp. 103-107, 2009.

[81] K. Araki, A. P. Turner, V. O. Shaffer et al., "mTOR regulates memory CD8 T-cell differentiation," Nature, vol. 460, no. 7251, pp. 108-112, 2009.

[82] C. Herbel, N. Patsoukis, K. Bardhan, P. Seth, J. D. Weaver, and V. A. Boussiotis, "Clinical significance of T cell metabolic reprogramming in cancer," Clinical and Translational Medicine, vol. 5, no. 1, p. 29, 2016.

[83] V. De Rosa, M. Galgani, A. Porcellini et al., "Glycolysis controls the induction of human regulatory $\mathrm{T}$ cells by modulating the expression of FOXP3 exon 2 splicing variants," Nature Immunology, vol. 16, no. 11, pp. 1174-1184, 2015.

[84] V. A. Gerriets, R. J. Kishton, M. O. Johnson et al., "Foxp3 and Toll-like receptor signaling balance Treg cell anabolic metabolism for suppression," Nature Immunology, vol. 17, no. 12, pp. 1459-1466, 2016.

[85] G. Andrejeva and J. C. Rathmell, "Similarities and distinctions of cancer and immune metabolism in inflammation and tumors," Cell Metabolism, vol. 26, no. 1, pp. 49-70, 2017.

[86] X. Yu and S. Li, "Non-metabolic functions of glycolytic enzymes in tumorigenesis," Oncogene, vol. 36, no. 19, pp. 2629-2636, 2017.

[87] J. G. Pastorino and J. B. Hoek, "Hexokinase II: the integration of energy metabolism and control of apoptosis," 
Current Medicinal Chemistry, vol. 10, no. 16, pp. 15351551, 2003.

[88] A. Haga, T. Funasaka, Y. Niinaka, A. Raz, and H. Nagase, "Autocrine motility factor signaling induces tumor apoptotic resistance by regulations Apaf- 1 and Caspase- 9 apoptosome expression," International Journal of Cancer, vol. 107, no. 5, pp. 707-714, 2003.

[89] T. Yanagawa, T. Funasaka, S. Tsutsumi, H. Watanabe, and A. Raz, "Novel roles of the autocrine motility factor/phosphoglucose isomerase in tumor malignancy," EndocrineRelated Cancer, vol. 11, no. 4, pp. 749-759, 2004.

[90] A. Tarze, A. Deniaud, M. Le Bras et al., "GAPDH, a novel regulator of the pro-apoptotic mitochondrial membrane permeabilization," Oncogene, vol. 26, no. 18, pp. 2606-2620, 2007.

[91] J. W. Kim and C. V. Dang, "Multifaceted roles of glycolytic enzymes," Trends in Biochemical Sciences, vol. 30, no. 3, pp. 142-150, 2005.

[92] F. Grosse, H. P. Nasheuer, S. Scholtissek, and U. Schomburg, "Lactate dehydrogenase and glyceraldehyde-phosphate dehydrogenase are single-stranded DNA-binding proteins that affect the DNA-polymerase-alpha-primase complex," European Journal of Biochemistry, vol. 160, no. 3, pp. 459467, 1986.

[93] S. Choudhary, B. P. De, and A. K. Banerjee, "Specific phosphorylated forms of glyceraldehyde 3-phosphate dehydrogenase associate with human parainfluenza virus type 3 and inhibit viral transcription in vitro," Journal of Virology, vol. 74, no. 8, pp. 3634-3641, 2000.

[94] M. W. Hentze and T. Preiss, "The REM phase of gene regulation," Trends in Biochemical Sciences, vol. 35, no. 8, pp. 423426, 2010.

[95] A. Castello, M. W. Hentze, and T. Preiss, "Metabolic enzymes enjoying new partnerships as RNA-binding proteins," Trends in Endocrinology and Metabolism, vol. 26, no. 12, pp. $746-$ 757, 2015.

[96] A. Castello, B. Fischer, K. Eichelbaum et al., "Insights into RNA biology from an atlas of mammalian mRNA-binding proteins," Cell, vol. 149, no. 6, pp. 1393-1406, 2012.

[97] R. Mukhopadhyay, J. Jia, A. Arif, P. S. Ray, and P. L. Fox, "The GAIT system: a gatekeeper of inflammatory gene expression," Trends in Biochemical Sciences, vol. 34, no. 7, pp. 324-331, 2009.

[98] P. Sampath, B. Mazumder, V. Seshadri, and P. L. Fox, "Transcript-selective translational silencing by gamma interferon is directed by a novel structural element in the ceruloplasmin mRNA 3' untranslated region," Molecular and Cellular Biology, vol. 23, no. 5, pp. 1509-1519, 2003.

[99] M. N. Lee, S. H. Ha, J. Kim et al., "Glycolytic flux signals to mTOR through glyceraldehyde-3-phosphate dehydro genase-mediated regulation of Rheb," Molecular and Cellular Biology, vol. 29, no. 14, pp. 3991-4001, 2009.

[100] D. Anastasiou, Y. Yu, W. J. Israelsen et al., "Pyruvate kinase M2 activators promote tetramer formation and suppress tumorigenesis," Nature Chemical Biology, vol. 8, no. 10, pp. 839-847, 2012.

[101] J. C. Alves-Filho and E. M. Palsson-McDermott, "Pyruvate kinase M2: a potential target for regulating inflammation," Frontiers in Immunology, vol. 7, p. 145, 2016.

[102] T. Shirai, R. R. Nazarewicz, B. B. Wallis et al., "The glycolytic enzyme PKM2 bridges metabolic and inflammatory dysfunction in coronary artery disease," The Journal of Experimental Medicine, vol. 213, no. 3, pp. 337-354, 2016.

[103] P. C. Ho, J. D. Bihuniak, A. N. Macintyre et al., "Phosphoenolpyruvate is a metabolic checkpoint of anti-tumor T cell responses," Cell, vol. 162, no. 6, pp. 1217-1228, 2015.

[104] C. H. Chang, J. Qiu, D. O'Sullivan et al., "Metabolic competition in the tumor microenvironment is a driver of cancer progression," Cell, vol. 162, no. 6, pp. 1229-1241, 2015.

[105] H. F. Dvorak, "Tumors: wounds that do not heal-redux," Cancer Immunology Research, vol. 3, no. 1, pp. 1-11, 2015.

[106] M. Sukumar, R. Roychoudhuri, and N. P. Restifo, "Nutrient competition: a new axis of tumor immunosuppression," Cell, vol. 162, no. 6, pp. 1206-1208, 2015.

[107] P. C. Ho and P. S. Liu, "Metabolic communication in tumors: a new layer of immunoregulation for immune evasion," Journal for ImmunoTherapy of Cancer, vol. 4, no. 1, p. 4, 2016.

[108] T. T. Vellinga, T. Borovski, V. C. de Boer et al., "SIRT1/ PGC1 $\alpha$-dependent increase in oxidative phosphorylation supports chemotherapy resistance of colon cancer," Clinical Cancer Research, vol. 21, no. 12, pp. 2870-2879, 2015.

[109] M. Z. Noman, G. Desantis, B. Janji et al., "PD-L1 is a novel direct target of HIF- $1 \alpha$, and its blockade under hypoxia enhanced MDSC-mediated T cell activation," The Journal of Experimental Medicine, vol. 211, no. 5, pp. 781-790, 2014.

[110] N. Patsoukis, K. Bardhan, P. Chatterjee et al., "PD-1 alters Tcell metabolic reprogramming by inhibiting glycolysis and promoting lipolysis and fatty acid oxidation," Nature Communications, vol. 6, p. 6692, 2015.

[111] S. R. Gordon, R. L. Maute, B. W. Dulken et al., "PD-1 expression by tumour-associated macrophages inhibits phagocytosis and tumour immunity," Nature, vol. 545, no. 7655, pp. 495-499, 2017.

[112] J. Kim, J. Kim, and J. S. Bae, "ROS homeostasis and metabolism: a critical liaison for cancer therapy," Experimental \& Molecular Medicine, vol. 48, no. 11, article e269, 2016.

[113] C. L. He, Y. Y. Bian, Y. Xue et al., "Pyruvate kinase M2 activates mTORC1 by phosphorylating AKT1S1," Scientific Reports, vol. 6, no. 1, p. 21524, 2016.

[114] E. M. Palsson-McDermott, A. M. Curtis, G. Goel et al., "Pyruvate kinase M2 regulates Hif-1alpha activity and IL-1beta induction and is a critical determinant of the warburg effect in LPS-activated macrophages," Cell Metabolism, vol. 21, no. 1, pp. 65-80, 2015.

[115] M. Goodman, Z. Liu, P. Zhu, and J. Li, "AMPK activators as a drug for diabetes, cancer and cardiovascular disease," Pharmaceutical Regulatory Affairs: Open Access, vol. 03, no. 02, 2014.

[116] M. Thurnher and G. Gruenbacher, "T lymphocyte regulation by mevalonate metabolism," Science Signaling, vol. 8, no. 370, p. re4, 2015.

[117] G. Sorrentino, N. Ruggeri, V. Specchia et al., "Metabolic control of YAP and TAZ by the mevalonate pathway," Nature Cell Biology, vol. 16, no. 4, pp. 357-366, 2014.

[118] J. Bellmunt, T. Powles, and N. J. Vogelzang, "A review on the evolution of PD-1/PD-L1 immunotherapy for bladder cancer: the future is now," Cancer Treatment Reviews, vol. 54, pp. 58-67, 2017.

[119] A. Casazza, D. Laoui, M. Wenes et al., "Impeding macrophage entry into hypoxic tumor areas by Sema3A/Nrp1 signaling 
blockade inhibits angiogenesis and restores antitumor immunity," Cancer Cell, vol. 24, no. 6, pp. 695-709, 2013.

[120] K. Movahedi, D. Laoui, C. Gysemans et al., "Different tumor microenvironments contain functionally distinct subsets of macrophages derived from Ly6C(high) monocytes," Cancer Research, vol. 70, no. 14, pp. 5728-5739, 2010.

[121] B. Ruffell, N. I. Affara, and L. M. Coussens, "Differential macrophage programming in the tumor microenvironment," Trends in Immunology, vol. 33, no. 3, pp. 119-126, 2012. 


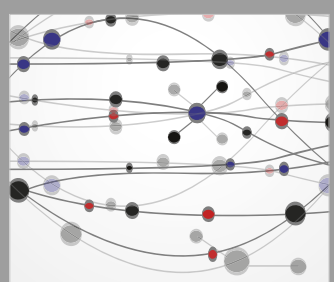

The Scientific World Journal
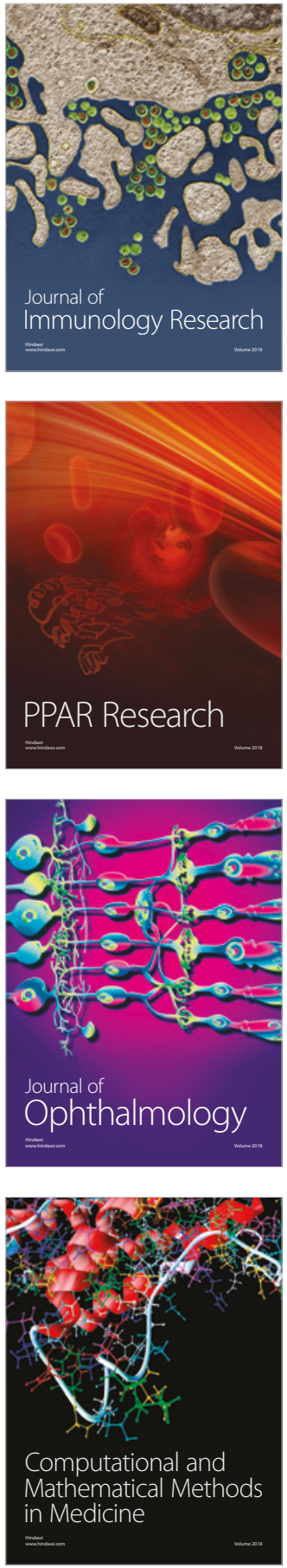

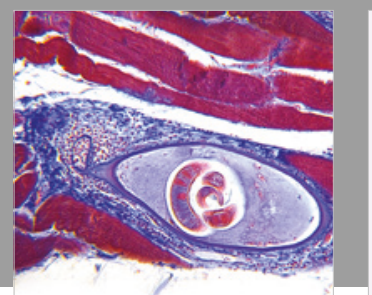

Gastroenterology Research and Practice

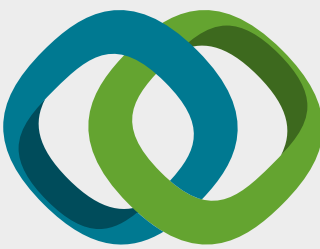

\section{Hindawi}

Submit your manuscripts at

www.hindawi.com
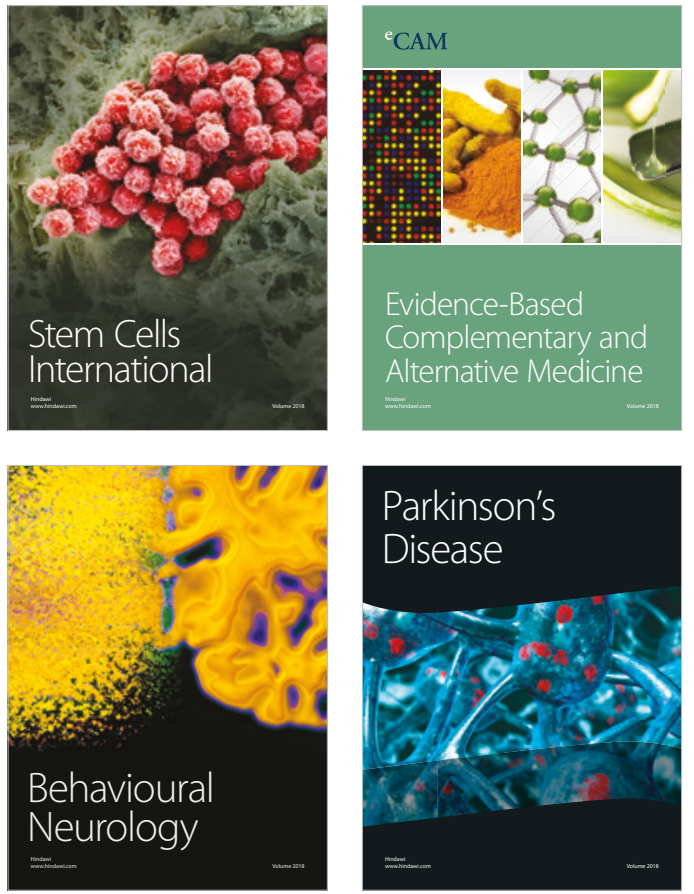

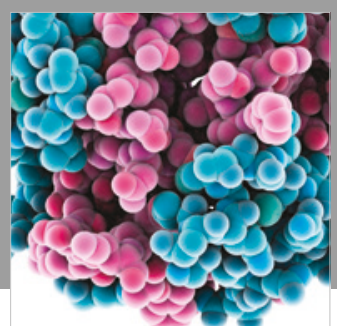

ournal of

Diabetes Research

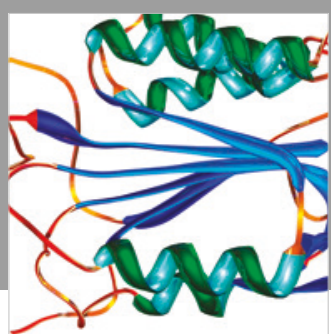

Disease Markers
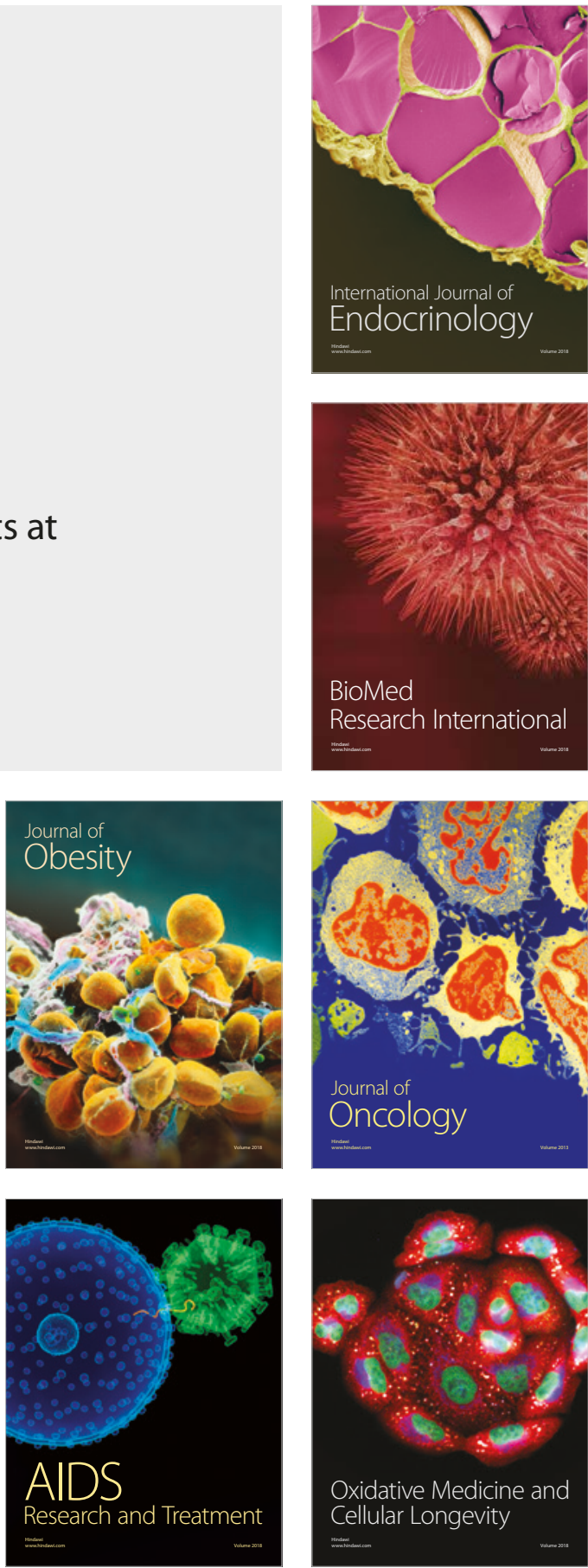